\title{
IMPACTO DE LAS TECNOLOGÍAS DE LA INFORMACIÓN Y LA COMUNICACIÓN EN LA JUVENTUD NAVARRA ${ }^{1}$
}

Concepción Naval y Sonia Lara

\section{RESUMEN}

La atención a la juventud, en todos sus aspectos, debe constituir uno de los pilares esenciales sobre los que asentar la construcción de una sociedad o de una comunidad. En buena parte, una comunidad se define por la valoración que haga de su propia juventud y la atención que le preste; aunque cabría decir que sobre todo se define por la valoración que la juventud hace de sí misma y por su proyección real de futuro.

En este escrito se presentan algunos datos preliminares del estudio Impacto de las Tecnologías de la Comunicación en la juventud navarra, 2001 (cfr. Naval y cols., 2002) que muestran las valoraciones que hacen los jóvenes de las tecnologías de la información y la comunicación (TIC). La cuestión planteada es: ¿cómo valoran los jóvenes la irrupción y el impacto de las nuevas tecnologías de la información en el entorno social, cultural y laboral? ¿Podemos detectar si la tan traída y llevada "fascinación tecnológica" es algo real y culturalmente significativo, o se circunscribe al ámbito de los «entendidos»? ¿Provocan

\footnotetext{
${ }^{1}$ Este escrito presenta algunos de los resultados de un estudio más amplio realizado en Navarra (España) con la esperanza de que pueda ser una experiencia provechosa en otros ámbitos geográficos, dada la extensión y difusión de estas tecnologías en el mundo entero (cfr. Naval y cols., 2002).
} 
las nuevas tecnologías procesos de aislamiento personal e insolidaridad o favorecen la comunicación interpersonal? ¿La posición ideológica determina de alguna manera el juicio que se forman los jóvenes sobre estas tecnologías de la comunicación? Los resultados de nuestros análisis pueden resultar en algún caso incluso sorprendentes, pero lo que delatan de modo manifiesto es la variedad de juicios; lo que hemos denominado pluridimensionalidad de las valoraciones, en las que las matizaciones, el reconocimiento implícito de la complejidad del fenómeno, el carácter imprevisible de su desarrollo, se imbrica muy probablemente con un estado de opinión germinal y poco estructurado todavía.

\section{INTRODUCCIÓN}

Uno de los objetivos principales al abordar el presente estudio fue el análisis de las valoraciones que jóvenes navarros hacen de las nuevas tecnologías. Nos planteamos, de hecho, estudiar las posibles mitologías establecidas sobre las Tecnologías de la Información y la Comunicación (TIC) ${ }^{2}$, a la vista del fuerte impacto que podían haber producido entre el sector más joven de la sociedad, su posible indefensión por falta de elementos de contraste derivados de la poca experiencia acumulada y la tendencia propia de la edad a la magnificación de los acontecimientos y las valoraciones.

¿Las TIC constituyen una panacea, la clave de muchos de los remedios para los problemas más acuciantes de la humanidad? O bien, ¿las TIC son una auténtica caja de Pandora, de las que nos sobrevendrán males difícilmente previsibles y gobernables? O en su caso, ¿son únicamente útiles instrumentos con los que poder satisfacer las exigencias de la sociedad actual?

\footnotetext{
${ }^{2}$ A lo largo de todo este estudio hemos considerado como Tecnologías de la Información y la Comunicación (TIC): ordenador personal; CD-ROM; Internet; correo electrónico; vídeo; TV Interactiva; teletexto; vídeo consola; TV de pago; TV por cable; antena parabólica; teléfono móvil; Fax; DVD; impresora y agenda electrónica.
} 
Para abordar esta cuestión no contamos de momento con estudios globales y mucho menos pormenorizados. Efectivamente, los estudios más habituales sobre las nuevas tecnologías se refieren fundamentalmente a la disponibilidad de tecnología y a su uso, dedicación y frecuencia. Sólo de modo indirecto y parcial encontramos referencias a las valoraciones que los jóvenes hacen de las TIC. Así, entre los estudios que podríamos destacar estarían: “óvenes españoles '99", Fundación Santa María, 1999; "Estudio vasco de Ciencia y Tecnología", Mikel Olazaran Rodríguez y Cristina Lavía Martínez, 2000; “Estadísticas Europeas sobre TIC", Revista Fuentes Estadísticas, 2001. Aquí se aprecia, efectivamente, la limitación en el tratamiento del tema, desde un punto de vista valorativo, que constituye uno de los puntos centrales de nuestro interés.

Para poder extraer conclusiones acerca de la valoración que los jóvenes de la Comunidad Foral de Navarra hacen de las TIC, se les pidió a los encuestados ${ }^{3}$ que, entre trece expresiones distintas, eligiesen aquellas que, en su opinión, mejor definen el papel de las nuevas tecnologías en la sociedad actual. Como vemos en la siguiente tabla, para cada uno de los items o expresiones más utilizadas para definir el papel de las TIC, los jóvenes encuestados podían elegir entre su exposición positiva, su exposición negativa, o sin darles en principio esa posibilidad, considerar que no estaban de acuerdo con ese tipo de definición o, bien, afirmar que tanto la opción positiva como la negativa podían ser posibles.

\footnotetext{
${ }^{3}$ El grupo de población que configura la muestra de este estudio es el de jóvenes navarros de entre 15 y 29 años ( $\mathrm{N}=117.905$ jóvenes). Este grupo representa el 22,5\% de la población total de esta comunidad autónoma. El muestreo realizado es aleatorio por cuotas establecidas ( $n=708$ jóvenes). El método de recogida de datos: entrevista personal por cuestionario estructurado.

Para la elaboración de este estudio se realizó un cuestionario base con 42 preguntas y 180 items, aplicado a un estudio orientativo de campo con 25 entrevistas. De este primer estudio se obtuvo el cuestionario final que por razones de operatividad y fiabilidad quedó reducido a 34 preguntas y 168 items. El trabajo de campo consistió en 708 entrevistas personalizadas.
} 
La comunicación está dividida en tres apartados. En el primero se exponen algunos de los principales resultados sobre la valoración que hacen los jóvenes navarros de las TIC clasificadas en varios grupos de opinión: valoración genérica positiva, influencia positiva en el trabajo, impacto en la sociabilidad, progresión y regresión, y valoración instrumental. En el segundo apartado se describen brevemente las características de los grupos de opinión descritos en el apartado anterior en función de la edad, sexo, nivel de estudios, etcétera. En el tercer y último apartado se apuntan algunas conclusiones sobre las valoraciones realizadas por los jóvenes navarros.

Tabla 1: ¿Cuál de las siguientes expresiones define mejor, a tu juicio, las nuevas tecnologías? (ver página 219).

\section{VALORACIÓN DE LOS JÓVENES DE LA COMUNIDAD FORAL NAVARRA DE LAS TIC}

A partir de las coincidencias registradas entre los jóvenes navarros a la hora de hacer una valoración sobre el impacto social, cultural o laboral de las nuevas tecnologías, detectamos cuatro grupos fundamentales que describimos a continuación.

\subsection{Valoración genérica positiva}

Nos encontramos, en primer lugar, con una coincidencia importante en las respuestas de los jóvenes en cuanto a ciertos aspectos que podemos considerar como positivos.

Se trata del grupo de jóvenes más numeroso, en torno al cincuenta por ciento (50\%) de los entrevistados que coinciden en la afirmación de que las TIC:

- Facilitan nuestro trabajo.

- Constituyen una etapa en el progreso general de la humanidad.

- Tienen una gran importancia para el futuro.

- Aumentan la comodidad en nuestra vida.

- Suponen una aportación positiva de conocimiento. 


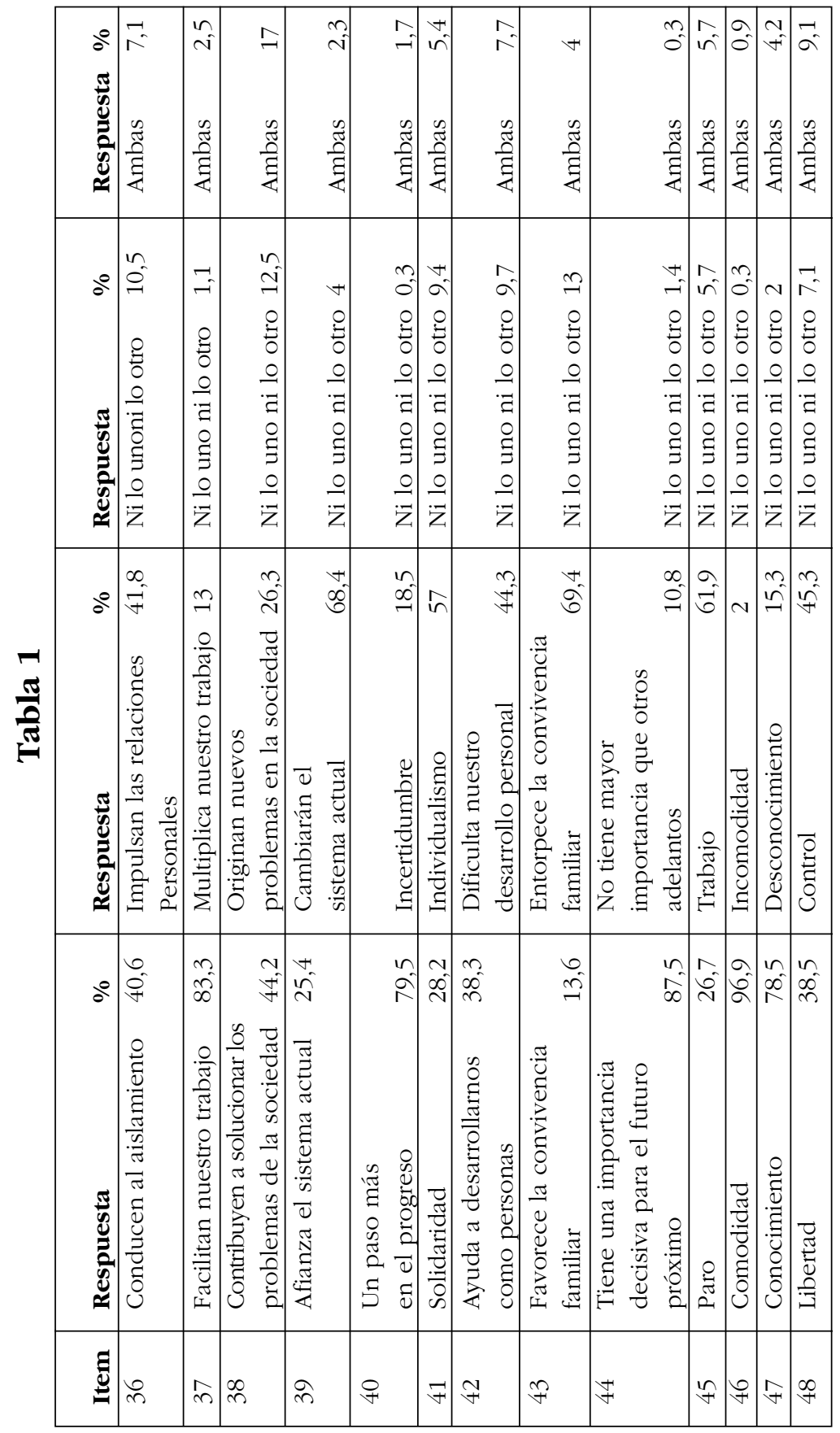


Lógicamente estamos ante una serie de afirmaciones reiteradas, que nos aportan específicamente el alto porcentaje de jóvenes que hace una valoración positiva de las nuevas tecnologías en lo referido a las condiciones de vida y bienestar (facilitan el trabajo y aportan comodidad), a lo que podríamos denominar su necesidad histórica (un paso más en el progreso y gran importancia para el futuro) y a la aportación de conocimientos.

Podemos afirmar, además, que esta apreciación global o genérica positiva se nos muestra bien asentada no sólo por el número de jóvenes que se incluyen bajo este factor de análisis sino también, por la coincidencia de las respuestas de los encuestados en cinco items bien diferenciados.

\subsection{Influencia positiva en el trabajo}

Relacionado con lo anterior, detectamos otro conjunto de jóvenes, también numeroso, directamente relacionado con las aportaciones positivas que las TIC están realizando en el ámbito laboral, según la opinión de la juventud navarra. A este grupo hemos decidido englobarlo bajo el epígrafe «influencia positiva en el trabajo" y alcanza casi al 52\% de la juventud.

Se trata de un conjunto de jóvenes que considera que en el mundo del trabajo y de la creación de empleo, la aportación de las nuevas tecnologías es francamente positiva por cuanto:

- Facilitan el trabajo.

- Incrementan nuestra comodidad.

- Contribuyen a la creación de empleo.

Nótese que el elemento diferenciador aquí respecto al grupo de jóvenes incluidos en el factor de análisis "visión genérica positiva" es referido a la creación de empleo.

Pues bien, no coinciden en el aspecto relacionado con el ámbito laboral, más de cien de los entrevistados que se mueven tanto en el grupo de "valoración genérica positiva" como en el de "influencia positiva en el trabajo". 
Entre estos dos factores de análisis, que cuentan con una proporción que es, en ambos casos, la mitad de la muestra utilizada para la realización de este estudio — -valoración genérica positiva": 50,2\% e "influencia positiva en el trabajo": $51,9 \%$ - existe una coincidencia que llega al 67\% de los componentes de ambos grupos. Esto se traduce en que la suma de jóvenes que hacen tanto una valoración positiva genérica de las TIC como de aquellos que realizan una valoración positiva referida únicamente al ámbito laboral, alcanzaría el 67\% indicado.

\subsection{Impacto en la sociabilidad}

Una vez analizados los cuatro items que nos permiten definir la valoración que hace la juventud navarra del "impacto en la sociabilidad" - preguntas 36, 41, 42, y 43 (ver Tabla 1) y tras entrecruzar las respuestas ofrecidas por los jóvenes, apreciamos que pueden establecerse dos grupos netamente diferenciados. Un grupo que alcanza al 18\% de los jóvenes y que configuran a aquellos que con rotundidad consideran que las nuevas tecnologías:

- Llevan al aislamiento.

- Conducen al individualismo.

- Entorpecen la convivencia familiar.

- Entorpecen nuestro desarrollo personal.

Y otro grupo que casi alcanza el 8\% de los jóvenes, quienes afirman que las nuevas tecnologías poseen un impacto positivo para el desarrollo de las relaciones sociales, en el más amplio sentido de esta expresión. Se trata de jóvenes que, para definir el impacto que las TIC ejercen en la sociedad, utilizan expresiones como éstas:

- Impulsan las relaciones personales.

- Conducen a la solidaridad.

- No entorpecen la convivencia familiar.

- Ayudan a desarrollarnos como personas. 


\subsection{Regresión y progresión}

Podemos diferenciar dos grupos diferentes de jóvenes: quienes consideran que las nuevas tecnologías suponen una regresión y, por otra parte, los que reafirman el sentido de progreso de las mismas.

\subsubsection{Regresión}

Aunque muchos de los encuestados que hayan proporcionado esa respuesta puedan referirse a la incertidumbre que cualquier proceso histórico conlleva, puede sin embargo detectarse una actitud netamente negativa — hipercrítica podríamos decirentre un reducido grupo de jóvenes. Son aquellos que:

- Consideran que las nuevas tecnologías implican «incertidumbre".

- Opinan que el panorama que nos abren en el orden socioeconómico es de "paro".

- Afirman que las nuevas tecnologías no contribuyen necesariamente a solucionar los problemas de la sociedad.

Podríamos considerar, globalmente, que para estos jóvenes las TIC representan más bien una regresión, un retroceso, y consideran que la aportación de las nuevas Tecnologías de la Información y de la Comunicación es claramente negativa en cuanto a la evolución social general, por más que podamos encontrar aspectos positivos en ellas, como los relativos a la comodidad y bienestar.

Este grupo, ciertamente minoritario, representa un 4\% de los jóvenes encuestados. Pero nos parece digno de ser reseñado, pese a lo exiguo de su número, por cuanto representa una clara opción de contestación o de confrontación directa con las nuevas tecnologías.

\subsubsection{Progresión}

Frente al anterior, encontramos un grupo más numeroso, que se mueve en torno al 13\% de los jóvenes encuestados, y que perciben las nuevas tecnologías con una óptica claramente opuesta. 
Es éste el porcentaje en el que se incluyen los jóvenes que afirman que las TIC:

- Son un paso más en el progreso.

- Inciden en la creación de empleo.

- Contribuyen a solucionar los problemas sociales.

- Permiten el desarrollo de las libertades individuales.

Como vemos, las tres primeras expresiones son exactamente las opuestas a las utilizadas por quienes opinan que las TIC implican una "regresión".

\subsection{Valoración instrumental}

Al grupo de jóvenes que optan por cualquiera de las dos respuestas secundarias, lo identificamos como «valoración instrumental", pues condicionan su valoración sobre el impacto de las TIC a la situación concreta y al tipo de uso dado a dichas tecnologías.

Pues bien, como observamos en la tabla, en siete de los items analizados el porcentaje de los que señalan «ni lo uno ni lo otro" o "ambas" supera el 10\% de los jóvenes encuestados.

- En el item 36, referido al aislamiento y las relaciones personales, el 17,6\% de los encuestados responde: "ambas" o "ni lo uno, ni lo otro".

- En el item 38, referido a los problemas sociales - el que registra una mayor valoración instrumental por parte de la juventud-, el 29,5\% de los jóvenes han optado por las dos respuestas secundarias.

- En el item que hace referencia a la solidaridad e individualismo (el número 41), el porcentaje de encuestados que ha respondido "ambas" o "ni lo uno, ni lo otro", asciende al 14,8\%.

- Con respecto al desarrollo personal, item número 42, el $17,4 \%$ de los encuestados ha optado por las dos respuestas secundarias.

- El 17\% de los jóvenes afirma que las nuevas tecnologías pueden "favorecer la convivencia familiar y entorpecerla" O "ninguna de las dos cosas". 
- En el item 45, el 11,4\% de los encuestados afirma que las nuevas tecnologías pueden "generar paro y también empleo" o "ni lo uno, ni lo otro".

- Por último, más del 16\% de los encuestados se acoge a las dos respuestas secundarias a la hora de opinar sobre la "libertad" o "control" que pueden generar las nuevas tecnologías.

A partir de todos estos datos, interpretamos que este tipo de respuestas indican, por una parte, una actitud en principio más reflexiva o que, al menos, parece apreciar más matices en la realidad que rodea a las TIC. Además de esta actitud, aparentemente más reflexiva, a lo que sí parecen apuntar ese tipo de respuestas es a una visión instrumental de las nuevas tecnologías.

Es decir, de la misma entidad de estos adelantos no se infiere una incidencia mecánica unidireccional, en determinados campos al menos. El valor positivo o negativo de las nuevas tecnologías dependerá del tipo de uso que los ciudadanos otorguen a la nuevas tecnologías en cada momento concreto.

Pues bien, interrelacionando y sumando el número de jóvenes que, ante diferentes items, optan por las dos respuestas secundarias, hemos descubierto la existencia de tres tipos diferentes de "valoración instrumental":

\subsubsection{Valoración instrumental referida a la «convivencia»}

Para detectar el grupo de jóvenes que hacen esta valoración instrumental, hemos tenido en cuenta los items 36 y 43 de la Tabla 1. A partir de dicho análisis, llegamos a la conclusión de que un total de 36 jóvenes afirman que las nuevas tecnologías "ni conducen al aislamiento, ni impulsan las relaciones personales" o "ambas", y, al mismo tiempo, opinan también que "ni favorecen la convivencia, ni la entorpecen" o "ambas cosas".

Es decir, el 5,1\% de los jóvenes encuestados no perciben una incidencia directa y unidireccional de las TIC en el desarrollo de las relaciones personales o familiares. 


\subsubsection{Valoración instrumental referida al «trabajo»}

En torno a un porcentaje semejante se mueve el grupo de jóvenes navarros que valoran del mismo modo las TIC respecto al trabajo (items 38 y 45 de la Tabla 1).

Para analizar el porcentaje de jóvenes navarros que hacen una valoración instrumental referida al trabajo hemos tomado como referencia los items 38 -que alude a la solución o generación de problemas sociales-y el 45 -que contrapone "paro" y "trabajo"—.

Tras dicho análisis, detectamos que existe un $6 \%$ de jóvenes que coinciden al afirmar que las TIC "ni solucionan, ni originan problemas sociales" o "ambas" y que, además, señalan que "ni inciden en el paro, ni en el trabajo" o, bien, pueden provocar "ambas" consecuencias.

\subsubsection{Valoración instrumental referida al «desarro- llo personal»}

El tercer grupo que detectamos, tras elaborar un análisis de las respuestas secundarias, es el formado por jóvenes que hacen una valoración instrumental referida al desarrollo personal. Para describir este grupo utilizamos los items 42 —referido a la ayuda o dificultad que las nuevas tecnologías aportan al desarrollo personal—y el número 48 —en el que se enfrentan los términos "libertad" $\mathrm{y}$ "Control"—.

Una vez analizadas las respuestas secundarias referidas a estos dos items, descubrimos que el 6,5\% de los jóvenes encuestados afirman que las TIC "ni ayudan, ni dificultan el desarrollo personal" o "ambas cosas a la vez" y, además, señalan que las TIC "ni suponen libertad, ni control" o bien, "ambas".

\section{CARACTERÍSTICAS DE LOS GRUPOS DE VALORACIÓN}

Analizamos las valoraciones hechas por los jóvenes navarros en función de diferentes características sociodemográficas (edad, sexo, nivel de estudios, etcétera) y nos parece de interés resaltar que: 
1.- Las valoraciones de los chicos suelen ser más radicales que las de las chicas. De este modo, los porcentajes masculinos suelen ser superiores a los femeninos a la hora de considerar a las TIC como "progreso", "regresión", etcétera.

2.- Es destacable el hecho de que el uso de Internet es inferior a la media entre el grupo de jóvenes que observan un "impacto positivo en la sociabilidad" y los que perciben las TIC como un elemento "regresivo". Pero, también desciende el uso de Internet, con respecto a la media, entre los jóvenes que consideran que las nuevas tecnologías son un elemento de "progreso". La poca cohesión entre estos datos parece ser, una vez más, un claro indicador de la enorme versatilidad de la materia que estamos analizando y, tal vez, de un cierto grado de desestructuración en la opinión sobre un fenómeno de tan reciente aparición.

3.- La posición ideológica tampoco parece ser un factor determinante entre los jóvenes navarros a la hora de hacer valoraciones sobre las TIC. La muestra confirma la no-alineación de las tendencias ideológicas con los grupos de opinión en referencia a las TIC. Se ve una manifestación de este hecho, por ejemplo, en cómo se hacen valoraciones entre los jóvenes que se sitúan en posiciones netas de izquierda (posiciones 1, 2 y 3 en un espectro de 1 a 10), en los que podemos suponer definiciones ideológicas más precisas.

4.- En cuanto a la edad, observamos que los jóvenes de 25 a 29 años suelen hacer unas valoraciones de las nuevas tecnologías, con tendencias más negativas y acentuando más su carácter instrumental que las realizadas por jóvenes de menor edad. Esta misma tendencia se observa también entre los jóvenes universitarios con respecto a jóvenes con estudios de nivel inferior.

5.- Se repite la pluridimensionalidad, al observar los resultados referidos a la situación profesional de la persona encuestada: tampoco ofrecen homogeneidad. Aunque, por los datos vistos hasta el momento, pudiese parecer que los jóvenes, 
mayores de 25 a 29 años de edad, valoran más negativamente las nuevas tecnologías, los datos referidos a la situación profesional, sin embargo, demuestran que el porcentaje de jóvenes que "Sólo trabajan" —que suelen ser los de mayor edad - y que "valoran positivamente la aportación de las TIC a la sociabilidad", supera ampliamente a la media.

El análisis del posicionamiento de los jóvenes ante las nuevas tecnologías, más allá de la constatación cuantitativa de la disponibilidad y uso de estos medios, nos muestra un panorama complejo que responde a la complejidad de las propias tecnologías, a la diversidad social e ideológica, y a la diversidad de los problemas que en estas valoraciones se abordan.

Por lo tanto, la búsqueda de las claves en la interpretación de los comportamientos, el diseño de las actuaciones pedagógicas, la resolución de las acciones públicas, no debe basarse en una petición de principios o en presupuestos de carácter dogmático, sino en la observación y el análisis de la complejidad de "lo que tenemos delante". La valoración que los jóvenes realizan sobre las nuevas tecnologías es un buen paradigma.

\section{A MODO DE SÍNTESIS}

Como reflexión final, señalaríamos que de los cuatro factores de análisis que describimos —-impacto en la sociabilidad", "valoración genérica positiva", "progreso y regresión" y «valoración instrumental»— tan sólo en dos de ellos detectamos dos grupos de jóvenes radical y absolutamente opuestos:

a) En el caso del factor de análisis "impacto en la sociabilidad", descubrimos un grupo que valora a las TIC por su "impacto positivo en la sociabilidad" y otro que, utilizando las mismas expresiones que el grupo anterior, pero en su exposición negativa, afirma el "impacto negativo de las nuevas tecnologías en la sociabilidad".

b) Lo mismo ocurría en el factor de análisis "progreso y regresión". Se confirmó la existencia de un grupo de jóvenes que consideran que las TIC representan progreso frente a otro 
grupo de encuestados que afirma el carácter regresivo de las mismas.

Tanto el primer caso — -impacto en la sociabilidad"— como el segundo — —progreso y regresión” — comparten el hecho de albergar interiormente dos grupos radicalmente opuestos entre sí. Dos grupos de jóvenes que tienen enfrente a otros dos grupos de personas encuestadas que, ante el mismo tipo de expresiones utilizadas para valorar las TIC, opinan exactamente lo contrario a ellos.

También nos gustaría destacar que, dentro de lo que denominamos "valoración genérica positiva", encontraremos prácticamente a todos aquellos jóvenes navarros que definen las TIC como factor de progreso y también a los que juzgan positivamente su impacto en la sociabilidad. Valoraciones que son perfectamente compatibles con una "visión genérica positiva".

Por el contrario, encontraremos ahí —entre los que hacen una valoración genérica positiva-, un número insignificante de quienes perciben la aportación de las nuevas tecnologías como regresiva. Pero todavía sorprende más que, entre el grupo de jóvenes que ofrecen una "visión genérica positiva", exista un número significativo —-más de un 30\%— de quienes consideran que su impacto en la sociabilidad es negativo.

La existencia de estas dos tendencias de opinión que, en principio, parecen oponerse a lo que hemos denominado como "valoración genérica positiva" nos lleva a concluir que, en este caso, existe un juicio ambivalente sobre las TIC. 


\section{BIBLIOGRAFÍA}

AAVV (2000). Estadísticas de la sociedad de la información. Cataluña 2000. Barcelona: Entitat Autònoma del Diari Oficial i de Publicacions (Catalunya).

AAVV (2000). La sociedad de la Información en España: situación actual y perspectivas 2000. Madrid: Telefónica I+D.

AAVV (2001). e-España 2001: informe anual sobre el desarrollo de la sociedad de la información en España. Madrid: Fundación Retevisión.

AAVV (2001). La sociedad de la información en el siglo XXI. Madrid: Proyectos y Producciones Editoriales Cyan, S.L.

AAVV (2001). La sociedad de la información en Iberoamérica 2001. Madrid: Asociación Hispana de Centros Investigación y Empresas Telecomunicaciones.

AIMC (2001). Información sobre la evolución de la audiencia de Internet en el estudio general de medios (EGM). Febreromarzo 1996 / octubre-noviembre 2001 [versión electrónica]. Asociación para la investigación de medios de comunicación. Accedido:http://www.aimc.es/aimc/html/inter/net.html (marzo, 2002).

Arbitron y Coleman (2000). The Broadband Revolution: How Superfast Internet Access Changes Media Habits in American Households [versión electrónica]. Accedido:

http://www.arbitron.com (2000).

Arbitron y Coleman (2001). Broadband Revolution 2: The Media World of Speedies, [versión electrónica] Accedido: http://www.arbitron.com (2000).

Arbitron y Edison Media Research (2000). Internet Study IV, The Buying Power of "Streamies", [versión electrónica] Accedido: http://www.arbitron.com (2000).

Arbitron y Edison Media Research (2000). Internet V, Startling New Insights about internet and Streaming, [versión electrónica] Accedido: http://www.arbitron.com (2000). 
Camacho, J.; Martín Serrano, M. y Velarde Hermida, O. (2000). Juventud Española, 2000. Estudio cuatrianual de la juventud española. [versión electrónica]. Instituto de la Juventud. Disponible: http://www.ugt.es/informes/injuve.pdf

Castells, M. (2001). Galaxia internet. Barcelona: Plaza \& Janés. CIS (1998, septiembre). Vida cotidiana de los jóvenes. Estudio CIS 2302 [versión electrónica]. http://www.cis.es/boletin/ 23/joven.htm (marzo, 2000).

CIS (2000, septiembre). Barómetro de septiembre 2000. Centro de Investigaciones Sociológicas. [versión electrónica]. http://www.aui.es/estadi/cis/cis.htm (marzo, 2000).

Codina, Mónica (ed.) (2001). De la ética desprotegida. Eunsa: Pamplona.

DEISS, Richard (2001). Estadísticas europeas sobre TIC's, Revista Fuentes Estadísticas, no 51.

ELZO, Javier y otros. (1999). Jóvenes Españoles 99. Fundación Santa María. Madrid.

EUSTAT (2001). La Sociedad de la Información y las familias. 2000. [versión electrónica]. Instituto Vasco de Estadística. Acceso: http://www.eustat.es/varios/informes/inf1758.pdf INJUVE (2001). Guía de consumo para la organización de actividades de tiempo libre. Madrid: Instituto de la Juventud (España).

INJUVE (2001). Informe juventud en España 2000. Madrid: Instituto de la Juventud (España).

Lucas Marín, A. (2000). La nueva sociedad de la información, una perspectiva desde Silicon Valley. Madrid: Editorial Trotta, S.A. Miguel, Amando de (2000). Dos generaciones de jóvenes de 1960-1998. Madrid: Instituto de la Juventud (España).

Morán Calvo-Sotelo, M. L. y Benedicto Millán, J. (2000). Jóvenes ciudadanos: propuesta para el análisis de las culturas ciudadanas de la juventud. Madrid: Instituto de la Juventud (España). 
Naval, C.; Lara, S.; Portilla, I. y Sádaba, Ch. (2002, en prensa). Impacto de las Tecnologías de la Comunicación en la juventud navarra, 2001. Gobierno de Navarra. Instituto Navarro de Deporte y Juventud: Pamplona.

NetValue (2000). El chat en España. La influencia de la cultura en el comportamiento de los Internautas. 2000 [versión electrónica]. Accedido: http://www.netvalue.com

NetValue (2000). Estudio europeo de la cobertura de los sitios de adultos. 2000 [versión electrónica]. Accedido: http://www.netvalue.com

NetValue (2000). Primeros datos acerca de internet en España. 2000 [versión electrónica]. Accedido: http://www.netvalue.com Olazaran, Mikel y otros (2000). Estudio vasco de Ciencia y Tecnología. Gobierno Vasco.

Telefónica I+D (2001, diciembre). La Sociedad de la Información en España. Perspectiva 2001-2005 [versión electrónica]. Informe elaborado por Telefónica. Accedido: http://www.tid.es/sociedad/si.htm (febrero, 2002).

US Department of Commerce (2002, febrero) A Nation Online: How Americans Are Expanding Their Use of the internet, Washintong DC, febrero de 2002 [versión electrónica]. US Department of Commerce Accedido: http://www.ntia.doc.gov/ ntiahome/dn/nationonline_020502.htm. (febrero, 2002).

Windham, L. y Orton, K. (2000). The Soul of the new consumer. The Attitudes, Behaviors, and Preferences of E-customers. Allworth Press: New York.

Zorogastua, J. (2001). Jóvenes 2001: la siguiente generación. Veintiuno: Revista de Pensamiento y Cultura, 50, 97-102. 